\title{
Planktonic algae and cyanoprokaryotes as indicators of ecosystem quality in the Mooi River system in the North-West Province, South Africa
}

\author{
A Venter ${ }^{1 *}$, S Barnard', MA Dickinson', S Janse van Vuuren ${ }^{1}$, A Levanets' ${ }^{1}$ and JC Taylor ${ }^{1,2}$ \\ 'Unit for Environmental Science and Management, North-West University, Potchefstroom 2520, South Africa \\ ${ }^{2}$ South African Institute for Aquatic Biodiversity, Grahamstown 6140, South Africa
}

\begin{abstract}
An ecologically healthy Mooi River system is important for maintaining the quality of potable water of Potchefstroom and surrounding areas. However, this system is under constant threat from anthropogenic pollution arising from both agricultural and mining activities in its catchment. A survey of planktonic algal and cyanoprokaryote assemblages in Klerkskraal, Boskop and Potchefstroom reservoirs was undertaken during 1999-2000 and 2010-2011. In all three dams, total algal and cyanoprokaryote concentrations were lower during the second survey (2010-2011), suggesting an improvement in ecosystem health. However, results also show a change from a Chrysophyceae-dominated community to one dominated by Bacillariophyceae. Increased numbers of diatom species that usually occur in eutrophic impoundments (Melosira varians, Cyclotella meneghiniana and Aulacoseira granulata) indicate an increase in the trophic status of the reservoirs, especially that of Boskop Dam, a trend mirrored by increases in conductivity as well as phosphorus and ammonium concentrations in all three reservoirs. It can therefore be concluded that although the ecosystem health of the Mooi River system is currently still good, further increases in nutrients such as phosphorus can cause proliferation of problem species (detected in enrichment cultures) and a deterioration of its water quality.
\end{abstract}

Keywords: Mooi River reservoirs, algal communities, cyanoprokaryotes, water quality

\section{INTRODUCTION}

The Mooi River originates in the Boons area and flows southwards through agricultural land into the Klerkskraal Dam, Boskop Dam and Potchefstroom Dam from where it meanders until it joins the Vaal River (Fig. 1). Other dams in the catchment of the Mooi River include Klipdrift Dam in the Loopspruit and Donaldson Dam in the Wonderfonteinspruit (Currie, 2001). The city of Potchefstroom gathers its potable water from surface- and groundwater in the Mooi River catchment. The water is collected and stored in the Boskop Dam from where it is transported in a $12-\mathrm{km}$ long uncovered cement canal to the water purification plant of the city (Annandale and Nealer, 2011).

Surface water quality in a region is largely determined both by natural processes and anthropogenic inputs (Kazi et al., 2009) and, in the case of the Mooi River system, anthropogenic inputs include agricultural as well as mining pollutants. The Mooi River is situated downstream of the current environmental crises on the West Rand and far West Rand regarding aspects such as acid mine drainage, closure of mines, and naturally rewatered gold mines which have negative effects on the Wonderfonteinspruit, as well as the underground located groundwater aquifers and

To whom all correspondence should be addressed.

III +27 18 299-2517; fax: +27 18 299-2370;

e-mail: 10066551@nwu.ac.za

Received 14 November 2012; accepted in revised form 7 October 2013.

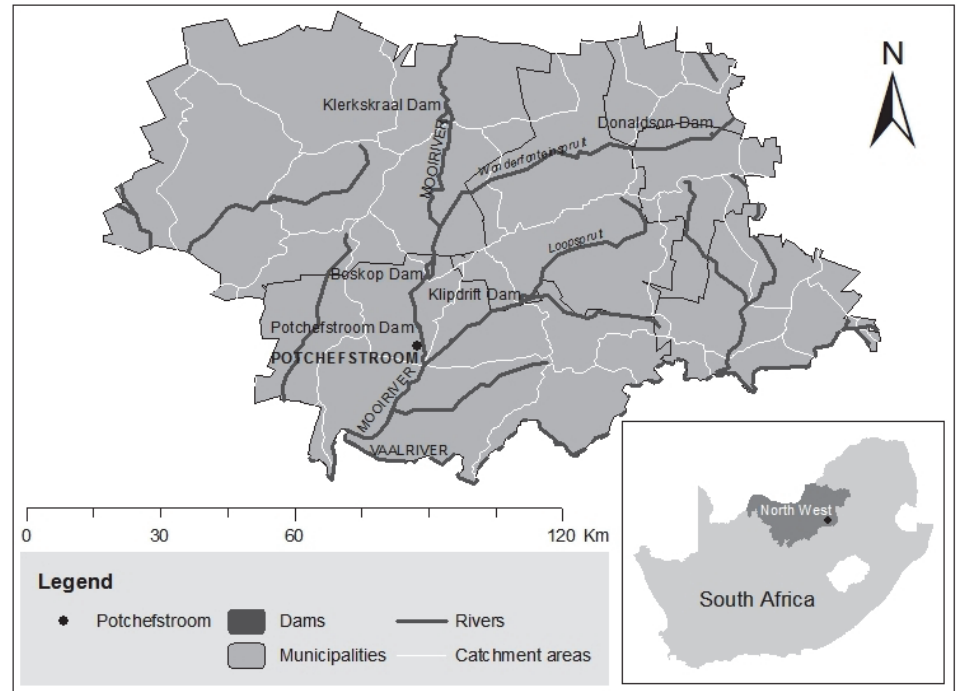

Figure 1

A map of the Mooi River System

springs in the karst landscape (Annandale and Nealer, 2011). During high rainfall conditions, Boskop and Potchefstroom dams receive water from the Mooirivierloop that is fed by water from the highly-polluted Wonderfonteinspruit. Although Klerkskraal Dam has no direct waterborne impacts from mining activity, windblown contamination from tailing storage facilities in the catchment is possible (Coetzee et al., 2006). The area surrounding the Mooi River, especially in the Boskop Dam area, has also been extensively surveyed for minerals, metals 
and other deposits and is therefore under constant threat from potential mining activity. Diamondiferous gravel diggings are already a common sight along the Mooi River between Klerkskraal Dam and the confluence with the Vaal River (Currie, 2001). As the Mooi River system is the main source of potable water for the University town of Potchefstroom and surrounding areas, deterioration in its water quality will impact a large number of people.

Biological communities reflect the overall ecological integrity by integrating various stressors, thus providing a broad measure of their synergistic impacts (De la Rey et al., 2004). Eutrophication is well known to affect planktonic autotroph abundance and composition. Phosphorus enrichment, in particular, often favours cyanophytes, including harmful toxinproducing taxa (Steinberg and Hartmann, 1988; O'Niel et al., 2012). These organisms have the potential to produce a variety of toxins that can be a health risk to humans and animals alike.

In 2004 the Department of Water Affairs and Forestry classified Boskop Dam as oligotrophic with very low algal productivity (Mogakabe, 2004); the aim of this study was to explore whether (and how) the phytoplankton communities and trophic status have changed in the past decade. During this study a survey of planktonic autotrophs of the dams in the Mooi River tributary was made, not only to determine if the health of the ecosystem has deteriorated over time, but also to serve as a baseline for future studies and environmental planning for the region.

\section{MATERIALS AND METHODS}

Water samples were collected on a monthly basis from May 1999 to July 2000 as well as from March 2010 until March 2011 at the wall of Klerkskraal Dam (S 26 $15^{\prime} 09.3^{\prime \prime}$ E $\left.27^{\circ} 09^{\prime} 34.1^{\prime \prime}\right)$, close to the main inflow of Boskop Dam (S 26 32' 43.6”'; E $27^{\circ}$ $\left.06^{\prime} 51.9^{\prime \prime}\right)$ and near the centre of Potchefstroom Dam (S $26^{\circ} 40^{\prime}$ $15.5^{\prime \prime}$; E $\left.27^{\circ} 05^{\prime} 38.7^{\prime \prime}\right)$. Samples were taken in the mornings, starting with Klerkskraal Dam and ending with Potchefstroom Dam. Water was sampled by lowering a bucket into the water, sampling water at about $20-30 \mathrm{~cm}$ and pouring it into $2-\ell$ plastic bottles. Samples were processed on the day of collection. On each sampling occasion physical parameters such as $\mathrm{pH}$, temperature (temp), conductivity (cond), turbidity (turb) and dissolved oxygen (LDO) were measured in situ at about $20 \mathrm{~cm}$ below the surface $w$ ith an YSI 556 MPS Multimeter.

The $2-\ell$ water samples collected from each reservoir were subdivided into samples for chemical analysis, chlorophyll- $a$ $(\mathrm{Chl} a)$ determination and algal identification. Chemical variables such as ammonium $\left(\mathrm{NH}_{4}\right)$, nitrate $\left(\mathrm{NO}_{3}\right)$, nitrite $\left(\mathrm{NO}_{2}\right)$ and orthophosphate $\left(\mathrm{PO}_{4}\right)$ were measured with a Palintest 8000 photometer.

Chlorophyll- $a$ concentration was determined with the method described by Sartory (1982) and Swanepoel et al. (2008). Two hundred (200) $\mathrm{m} \ell$ water was filtered through a Whatman GF/C filter. The chlorophyll gathered on the filter was extracted with $10 \mathrm{~m} \ell 95 \%$ ethanol in a water bath at $78^{\circ} \mathrm{C}$ for $5 \mathrm{~min}$. The samples were removed and left in the dark to cool down. The difference in absorbance of the extract was determined at 665 and $750 \mathrm{~nm}$ respectively, using 95\% ethanol as the blank. The difference in absorbance of the same sample was again determined $2 \mathrm{~min}$ after acidification with $0.1 \mathrm{~m} \ell 1$ $\mathrm{N} \mathrm{HCl}$. The chlorophyll- $a$ concentration was calculated with the following equation: chlorophyll- $a\left(\mu \mathrm{g} \cdot \ell^{-1}\right)=[(\mathrm{A} 665-\mathrm{A} 750)$ - (A665 -A750 $)$ x 28.66 x extract volume]/volume of sample, where A665 and A665 (and A750, A750 ) represent absorbance measured at 665 (and 750) nm before and after acidification.

Phytoplankton samples were preserved in $2 \%$ formaldehyde (final concentration) immediately after collection. Despite the fact that formalin poses a health hazard, as well as sometimes causing changes in cell dimensions, damage and distortion of chloroplasts, it remains the most commonly used liquid preservative (John et al., 2002). Concentrations of $2.5 \%$ are less damaging than higher concentration ranges in the order of $4 \%$ (John et al., 2002). Formaldehyde was preferred to Lugol's solution because the latter often discolours the cell contents, which must be clearly visible for correct identification. Phytoplankton identification and enumeration were done according to the sedimentation technique using gravity as described in Utermöhl (1958) and Swanepoel et al. (2008). Gas vacuoles of cyanoprokaryotes were pressure-deflated in a special container using a mechanical hammer. Up to $5 \mathrm{~m} \ell$ (depending on the density of the algae) was then pipetted into sedimentation tubes. The sedimentation tubes were filled with distilled water and covered with circular glass coverslips. The sedimentation tubes were left for a period of at least 2 days in a desiccator in order to allow the cells to settle. Algae and cyanoprokaryotes were identified and counted using an inverted microscope. Identification and enumeration was done by the same analyst to ensure comparability between the two periods. Literature used for identification were Croasdale et al. (1994); Ettl et al. (1999); Hindak (2008); Huber-Pestalozzi (1961); John et al. (2002); Komárek and Anagnostidis (2005); Taylor et al. (2007a); Wehr and Sheath (2003) and Oyadomari (2001).

An aliquot of $50 \mathrm{~m} \ell$ from each sampling site was enriched with 100 ml GBG11 growth medium (Krüger, 1978) and incubated at a temperature of $20^{\circ} \mathrm{C}$ and a continuous light intensity of $15 \mu \mathrm{mol} \mathrm{m} \mathrm{m}^{-2} \mathrm{~s}^{-1}$ to stimulate the growth of algae and cyanoprokaryotes present in low concentrations. Enrichment studies were not done during the first survey.

The survey done from March 2010 to March 2011 (hereafter referred to as current survey) and the survey from May 1999 to July 2000 (hereafter referred to as previous survey) were done at the same localities using the same methods and supervised by the same person. Differences in algal and cyanoprokaryote composition, as well as in physical and chemical variables between samples collected during the current and previous surveys were explored and tested using Statistica version 10 software (StatSoft Inc.). The Kolmogorov-Smirnov and Lilliefors test for normality was used to determine if the variables were distributed parametrically. The data did not meet the assumptions of normality in the distribution of all variables. The Kruskal-Wallis ANOVA for non-parametric data was used for comparing multiple independent samples to determine differences between the variables in each reservoir, as well as between variables from the two time periods. CANOCO version 4.5 software was used to perform multivariate and ordination analyses (Ter Braak and Smilauer, 1998). Only the datasets that contained all the variables were used for multivariate analysis.

\section{RESULTS}

\section{Community composition}

A species list of cyanoprokaryotes and algal taxa was compiled for each impoundment to examine any changes in the 10-year interval between the previous and current surveys (Table 1).

In Klerkskraal Dam, 4 Cyanophyceae species occurred during both periods. Diatoms increased from 10 to 15 species, 
TABLE 1

Comparison of the species composition between the two sampling periods

(1999-2000 and 2010-2011) in the three dams located on the Mooi River

\begin{tabular}{|c|c|c|c|c|c|c|c|}
\hline & Klerkskraal Dam & \multicolumn{2}{|c|}{ Boskop Dam } & Potchefstroom Dam \\
\cline { 2 - 6 } & $\begin{array}{c}1999- \\
2010-\end{array}$ & $\begin{array}{c}1999- \\
2010-\end{array}$ & $\begin{array}{c}1999- \\
2090- \\
2011\end{array}$ & $\begin{array}{c}2000- \\
2011\end{array}$ \\
\hline
\end{tabular}

\section{CYANOPHYCEAE}

\begin{tabular}{|l|l|l|l|l|l|l|l|l}
2000 & 2011 & 2000 & 2011 & 2000 & 2011 \\
\hline
\end{tabular}

Arthrospira sp.

Cyanobacterium sp.

Cylindrospermopsis raciborskii (Woloszynska) Seenayya et Subba Raju

Merismopedia minima Beck

Microcystis aeruginosa (Kützing) Kützing

Microcystis flos-aquae (Wittrock) Kirchner

Microcystis wesenbergii (Komárek) Komárek

Oscillatoria sp.

Oscillatoria simplicissima Gomont

Pseudanabaena sp.

Snowella sp.

Total number of Cyanophyceae species

Total number of Cyanophyceae species shared between surveys

\begin{tabular}{|c|c|c|c|c|c|}
\hline & & & & $\checkmark$ & $\checkmark$ \\
\hline & & & $\checkmark$ & & \\
\hline & & & $\checkmark$ & & \\
\hline & $\checkmark$ & $\checkmark$ & $\checkmark$ & $\checkmark$ & \\
\hline \multirow[t]{2}{*}{$\checkmark$} & $\checkmark$ & $\checkmark$ & $\checkmark$ & $\checkmark$ & $\checkmark$ \\
\hline & & & & & $\checkmark$ \\
\hline$\checkmark$ & & & $\checkmark$ & $\checkmark$ & \\
\hline \multirow[t]{2}{*}{$\checkmark$} & & & $\checkmark$ & $\checkmark$ & $\checkmark$ \\
\hline & & & & $\checkmark$ & \\
\hline \multirow[t]{2}{*}{$\checkmark$} & $\checkmark$ & $\checkmark$ & $\checkmark$ & $\checkmark$ & $\checkmark$ \\
\hline & $\checkmark$ & & $\checkmark$ & & $\checkmark$ \\
\hline 4 & 4 & 3 & 8 & 7 & 6 \\
\hline \multicolumn{2}{|c|}{2} & \multicolumn{2}{|c|}{3} & \multicolumn{2}{|c|}{4} \\
\hline
\end{tabular}

BACILLARIOPHYCEAE

Achnanthidium minutissimum (Kützing) Czarnecki

Amphipleura sp.

Aulacoseira granulata (Ehrenberg) Simonsen

Aulacoseira muzanensis (Meister) Krammer

Asterionella formosa Hassall

Cocconeis pediculus Ehrenberg

Cyclotella meneghiniana Kützing

Cymatopleura sp.

Cymbella spp.

Diadesmus confervacea Kützing (syn. Navicula confervacea (Kützing) Grunow in Van Heurck)

Diatoma vulgaris Bory

Epithemia sp.

Fragilaria ulna (Nitzsch) Lange-Bertalot

Gomphonema spp.

Gyrosigma sp.

Melosira varians C.Agardh

Navicula spp.

Nitzschia palea (Kützing) W.Smith

Nitzschia spp.

Pinnularia sp.

Pleurosigma sp.

Rhopaloidia sp.

Stephanodiscus spp.

Surirella sp.

Total number of Bacillariophyceae species

Total number of Bacillariophyceae species shared between surveys

CHLOROPHYCEAE

\begin{tabular}{|c|c|c|c|c|c|}
\hline & $\checkmark$ & $\checkmark$ & $\checkmark$ & & \\
\hline$\checkmark$ & $\checkmark$ & $\checkmark$ & $\checkmark$ & $\checkmark$ & $\checkmark$ \\
\hline$\checkmark$ & $\checkmark$ & & & & \\
\hline
\end{tabular}

Actinotaenium sp.

Ankistrodesmus sp.

Carteria sp.

Carteria simplicissima Pascher

Characium limneticum Lemmermann

Chlamydomonas incerta Pascher 


\begin{tabular}{|c|c|c|c|c|c|c|}
\hline Chlamydomonas bicocca Pascher & $\checkmark$ & $\checkmark$ & & & $\checkmark$ & \\
\hline Chlamydomonas conferta Korshikov & & & & & & $\checkmark$ \\
\hline Chlamydomonas sp. & $\checkmark$ & $\checkmark$ & $\checkmark$ & $\checkmark$ & $\checkmark$ & $\checkmark$ \\
\hline Chlorella sp. & $\checkmark$ & $\checkmark$ & $\checkmark$ & $\checkmark$ & $\checkmark$ & \\
\hline Chlorococcum infusionum (Schrank) Meneghini & $\checkmark$ & & $\checkmark$ & & $\checkmark$ & \\
\hline Chlorogonium sp. & $\checkmark$ & $\checkmark$ & & & & \\
\hline Closterium cornu Ehrenberg ex Ralfs & $\checkmark$ & $\checkmark$ & $\checkmark$ & & $\checkmark$ & $\checkmark$ \\
\hline Coelastrum pseudomicroporum Korshikov & $\checkmark$ & & $\checkmark$ & $\checkmark$ & $\checkmark$ & \\
\hline Coelastrum reticulatum (P.A.Dangeard) Senn & $\checkmark$ & & & & $\checkmark$ & \\
\hline Conococcus elongates H.J.Carter & $\checkmark$ & & & & & \\
\hline Cosmarium sp. & $\checkmark$ & & $\checkmark$ & $\checkmark$ & $\checkmark$ & $\checkmark$ \\
\hline Crucigenia fenestrate (Schmidle) Schmidle & & & & & $\checkmark$ & \\
\hline Crucigenia lauterbornii (Schmidle) Schmidle & $\checkmark$ & & $\checkmark$ & & $\checkmark$ & \\
\hline Crucigenia tetrapedia (Kirchner) Kuntze & $\checkmark$ & & $\checkmark$ & $\checkmark$ & $\checkmark$ & $\checkmark$ \\
\hline Crucigeniella rectangularis (Nägeli) Komárek & $\checkmark$ & & $\checkmark$ & & $\checkmark$ & \\
\hline Dictyosphaerium elegans Bachmann & & & & & $\checkmark$ & \\
\hline Golenkinia radiata Chodat & $\checkmark$ & $\checkmark$ & $\checkmark$ & $\checkmark$ & $\checkmark$ & $\checkmark$ \\
\hline Gonatozygon sp. & & $\checkmark$ & & $\checkmark$ & & $\checkmark$ \\
\hline Kirchneriella sp. & $\checkmark$ & $\checkmark$ & $\checkmark$ & $\checkmark$ & $\checkmark$ & \\
\hline Lagerheimia balatonica (Scherffel) Hindák & & & $\checkmark$ & & & \\
\hline Lagerheimia chodatii C.Bernard & $\checkmark$ & & $\checkmark$ & & $\checkmark$ & \\
\hline Lagerheimia longiseta (Lemmermann) Printz & & & $\checkmark$ & & & \\
\hline Micractinium sp. & & & & & $\checkmark$ & \\
\hline Monoraphidium arcuatum (Korshikov) Hindák & & $\checkmark$ & & & $\checkmark$ & \\
\hline Monoraphidium circinale (Nygaard) Nygaard & $\checkmark$ & & & $\checkmark$ & $\checkmark$ & $\checkmark$ \\
\hline Monoraphidium contortum (Thuret) Komàrková-Legnerová & $\checkmark$ & & & & & \\
\hline Monoraphidium minutum (Nägeli) Komárková-Legnerová & $\checkmark$ & & $\checkmark$ & $\checkmark$ & $\checkmark$ & $\checkmark$ \\
\hline Monoraphidium pseudobraunii (Belcher et Swale) Heynig & & & & & & $\checkmark$ \\
\hline Monoraphidium sp. & $\checkmark$ & $\checkmark$ & $\checkmark$ & $\checkmark$ & $\checkmark$ & $\checkmark$ \\
\hline Oocystis lacustris Chodat & $\checkmark$ & $\checkmark$ & $\checkmark$ & $\checkmark$ & $\checkmark$ & $\checkmark$ \\
\hline Oocystis marsonii Lemmermann & & & & & $\checkmark$ & \\
\hline Oocystis pusilla Hansgirg & & & & & $\checkmark$ & $\checkmark$ \\
\hline Oocystis sp. & $\checkmark$ & $\checkmark$ & $\checkmark$ & $\checkmark$ & & \\
\hline Pandorina morum (O.F.Müller) Bory de Saint-Vincent & & $\checkmark$ & $\checkmark$ & $\checkmark$ & $\checkmark$ & $\checkmark$ \\
\hline Pediastrum duplex Meyen & $\checkmark$ & & $\checkmark$ & & $\checkmark$ & $\checkmark$ \\
\hline Pediastrum simplex Meyen & $\checkmark$ & $\checkmark$ & $\checkmark$ & $\checkmark$ & $\checkmark$ & \\
\hline Pediastrum tetras (Ehrenberg) Ralfs & $\checkmark$ & $\checkmark$ & $\checkmark$ & $\checkmark$ & $\checkmark$ & $\checkmark$ \\
\hline Phacotus lenticularis (Ehrenberg) Stein & $\checkmark$ & & $\checkmark$ & & $\checkmark$ & \\
\hline Pteromonas angulosa Lemmermann & $\checkmark$ & & & & & \\
\hline Scenedesmus abundans (O. Kirchner) Chodat & & & & $\checkmark$ & & $\checkmark$ \\
\hline Scenedesmus acuminatus (Lagerheim) Chodat & & $\checkmark$ & & & & \\
\hline Scenedesmus disciformis (Chodat) Fott et Komárek & & $\checkmark$ & $\checkmark$ & $\checkmark$ & $\checkmark$ & $\checkmark$ \\
\hline Scenedesmus lefevrii Komárek & & $\checkmark$ & $\checkmark$ & $\checkmark$ & $\checkmark$ & $\checkmark$ \\
\hline Scenedesmus quadricauda Chodat & $\checkmark$ & $\checkmark$ & $\checkmark$ & $\checkmark$ & $\checkmark$ & $\checkmark$ \\
\hline Scenedesmus sp. & $\checkmark$ & $\checkmark$ & $\checkmark$ & $\checkmark$ & $\checkmark$ & $\checkmark$ \\
\hline Sphaerocystis planctonica R. Chodat & & & & & $\checkmark$ & \\
\hline Sphaerocystis schroeteri Chodat & $\checkmark$ & $\checkmark$ & & & $\checkmark$ & $\checkmark$ \\
\hline Staurastrum sp. & $\checkmark$ & $\checkmark$ & $\checkmark$ & $\checkmark$ & $\checkmark$ & \\
\hline Tetraedron caudatum (Corda) Hansgirg & & & & $\checkmark$ & & $\checkmark$ \\
\hline Tetraedron mediocris Hindák & & & & & & $\checkmark$ \\
\hline Tetraedron minimum (A.Braun) Hansgirg & $\checkmark$ & & $\checkmark$ & $\checkmark$ & $\checkmark$ & $\checkmark$ \\
\hline Tetraedron sp. & $\checkmark$ & & & $\checkmark$ & & $\checkmark$ \\
\hline Tetrastrum komarekii Hindák & & $\checkmark$ & $\checkmark$ & $\checkmark$ & $\checkmark$ & $\checkmark$ \\
\hline Tetrastrum staurogeniaeforme (Schröder) Lemmermann & & & & $\checkmark$ & $\checkmark$ & \\
\hline
\end{tabular}




\begin{tabular}{|c|c|c|c|c|c|c|}
\hline Volvox sp. & $\checkmark$ & & & & $\checkmark$ & \\
\hline Total number of Chlorophyceae species & 36 & 24 & 34 & 28 & 44 & 29 \\
\hline Total number of Chlorophyceae species shared between surveys & \multicolumn{2}{|c|}{17} & \multicolumn{2}{|c|}{21} & \multicolumn{2}{|c|}{22} \\
\hline \multicolumn{7}{|l|}{ CRYPTOPHYCEAE } \\
\hline Cryptomonas major Butcher & $\checkmark$ & $\checkmark$ & $\checkmark$ & $\checkmark$ & $\checkmark$ & $\checkmark$ \\
\hline Cryptomonas minor J.Schiller & $\checkmark$ & $\checkmark$ & $\checkmark$ & $\checkmark$ & $\checkmark$ & $\checkmark$ \\
\hline $\begin{array}{l}\text { Rhodomonas lacustris Pascher et Ruttner var. nannoplanctica (Skuja) } \\
\text { Javornicky }\end{array}$ & $\checkmark$ & $\checkmark$ & $\checkmark$ & & $\checkmark$ & \\
\hline Total number of Cryptophyceae species & 3 & 3 & 3 & 2 & 3 & 2 \\
\hline Total number of Cryptophyceae shared between surveys & \multicolumn{2}{|c|}{3} & \multicolumn{2}{|c|}{2} & \multicolumn{2}{|c|}{2} \\
\hline \multicolumn{7}{|l|}{ CHRYSOPHYCEAE } \\
\hline Dinobryon sp. (shared between surveys) & $\checkmark$ & $\checkmark$ & $\checkmark$ & $\checkmark$ & $\checkmark$ & $\checkmark$ \\
\hline Total number of Chrysophyceae species & 1 & 1 & 1 & 1 & 1 & 1 \\
\hline \multicolumn{7}{|l|}{ DINOPHYCEAE } \\
\hline Ceratium hirundinella (O.F.Müller) Dujardin & & & $\checkmark$ & $\checkmark$ & $\checkmark$ & $\checkmark$ \\
\hline Peridinium sp. & $\checkmark$ & & $\checkmark$ & $\checkmark$ & $\checkmark$ & $\checkmark$ \\
\hline Peridinium gatunense Nygaard & & $\checkmark$ & & & & \\
\hline Sphaerodinium sp. & $\checkmark$ & $\checkmark$ & $\checkmark$ & $\checkmark$ & $\checkmark$ & $\checkmark$ \\
\hline Total number of Dinophyceae species & 2 & 2 & 3 & 3 & 3 & 3 \\
\hline Total number of Dinophyceae species shared between surveys & \multicolumn{2}{|c|}{1} & \multicolumn{2}{|c|}{3} & \multicolumn{2}{|c|}{3} \\
\hline \multicolumn{7}{|l|}{ EUGLENOPHYCEAE } \\
\hline Euglena hemichromata Skuja & & & & & $\checkmark$ & \\
\hline Euglena pusilla Playfair & & & $\checkmark$ & & & \\
\hline Euglena sp. & $\checkmark$ & $\checkmark$ & & $\checkmark$ & $\checkmark$ & $\checkmark$ \\
\hline Phacus acuminatus Stokes & & & $\checkmark$ & & $\checkmark$ & \\
\hline Phacus meson Pochmann & $\checkmark$ & $\checkmark$ & $\checkmark$ & & $\checkmark$ & $\checkmark$ \\
\hline Strombomonas fluviatilis (Lemmermann) Deflandre & & & & & $\checkmark$ & \\
\hline Strombomonas jaculata (Palmer) Deflandre & $\checkmark$ & $\checkmark$ & & $\checkmark$ & & $\checkmark$ \\
\hline Strombomonas ovalis (Playfair) Deflandre & & $\checkmark$ & $\checkmark$ & $\checkmark$ & & $\checkmark$ \\
\hline Trachelomonas hispida (Perty) F. Stein & & $\checkmark$ & & $\checkmark$ & & \\
\hline Trachelomonas intermedia P.A. Dangeard & $\checkmark$ & $\checkmark$ & $\checkmark$ & $\checkmark$ & $\checkmark$ & $\checkmark$ \\
\hline Trachelomonas volvocina (Ehrenberg) Ehrenberg & $\checkmark$ & $\checkmark$ & $\checkmark$ & $\checkmark$ & $\checkmark$ & $\checkmark$ \\
\hline Total number of Euglenophyceae species & 5 & 7 & 6 & 6 & 7 & 6 \\
\hline Total number of Euglenophyceae species shared between surveys & \multicolumn{2}{|c|}{5} & \multicolumn{2}{|c|}{3} & \multicolumn{2}{|c|}{4} \\
\hline TOTAL PHYTOPLANKTON SPECIES RICHNESS & 61 & 56 & 62 & 64 & 76 & 67 \\
\hline
\end{tabular}

whereas Chlorophycean species declined from 36 to 24 . The number of species of Cryptophyceae (3), Chrysophyceae (1) and Dinophyceae (2) did not change, but Euglenophyceae increased slightly from 5 to 7 . Total phytoplankton species richness in Klerkskraal Dam decreased from 61 to 56 during the decade.

In Boskop Dam, increases in species number of

Cyanophyceae and Bacillariophyceae were observed ( 3 to 8 and 12 to 16 , respectively), while species richness of Chlorophyceae and Cryptophyceae decreased (34 to 28 , and 3 to 2, respectively). The number of Chrysophyceae (1), Dinophyceae (3) and Euglenophyceae (6) species remained constant. Overall phytoplankton species richness in Boskop Dam increased from 62 to 64 species.

In Potchefstroom Dam, Cyanophyceae species decreased from 7 to 6 , but as with the other 2 dams, species numbers of Bacillariophyceae increased from 11 to 20 and Chlorophyceae species decreased from 44 to 29. Numbers of Cryptophyceae and Euglenophyceae species decreased slightly from 3 to 2 and 7 to 6, respectively, while numbers of the Dinophyceae (3) and
Chrysophyceae (1) remained constant (Table 1). Total phytoplankton species richness decreased from the previous (76 species) to the current survey ( 67 species) by 9 species.

During the previous survey, species richness differed slightly in Klerkskraal (61 species) and Boskop Dams (62 species) but increased to 76 species in the downstream Potchefstroom Dam. During the present survey, downstream increases in species richness were evident down the entire reservoir cascade (Klerkskraal Dam - 56, Boskop Dam - 64 and Potchefstroom Dam - 67 species).

The current survey shows that several species, absent during the previous survey, now occur in all three impoundments. Examples are the cyanobacterium Snowella; the green alga Gonatozygon as well as genera from the Bacillariophyceae, namely Diatoma vulgaris Bory (typical of eutrophic waters) and Gomphonema species. Conversely, several species of Chlorophyceae (Crucigenia lauterbornii, Crucigeniella rectangularis, Lagerheimia codatii and Phacotus lenticularis) disappeared. Enrichment experiments (Table 2) also demonstrated 


\begin{tabular}{|c|c|c|c|}
\hline \multicolumn{4}{|c|}{$\begin{array}{c}\text { TABLE } 2 \\
\text { Species list obtained from enriched samples from March 2010 - March } 2011\end{array}$} \\
\hline & Klerkskraal Dam & Boskop Dam & Potchefstroom Dam \\
\hline \multicolumn{4}{|l|}{ CYANOPHYCEAE } \\
\hline Anabaena sp. & & $\checkmark$ & $\checkmark$ \\
\hline Aphanocapsa sp. & $\checkmark$ & $\checkmark$ & $\checkmark$ \\
\hline Aphanothece flocossa (Zalessky) G. Cronberg et Komárek & $\checkmark$ & $\checkmark$ & \\
\hline Calothrix sp. & & & $\checkmark$ \\
\hline Cyanosarcina sp. & & $\checkmark$ & \\
\hline Geitlerinema amphibium (C. Agardh ex Gomont) Anagnostidis & $\checkmark$ & $\checkmark$ & $\checkmark$ \\
\hline Leptolyngbya sp. & $\checkmark$ & $\checkmark$ & $\checkmark$ \\
\hline Lyngbya martensiana Meneghini ex Gomont & & & $\checkmark$ \\
\hline Merismopedia sp. & $\checkmark$ & $\checkmark$ & $\checkmark$ \\
\hline Microcystis sp. & & $\checkmark$ & $\checkmark$ \\
\hline Oscillatoria tenuis & $\checkmark$ & $\checkmark$ & \\
\hline Planktothrix sp. & & & $\checkmark$ \\
\hline Phormidium aerugineo-caeruleum (Gomont) Anagnostidis & & & $\checkmark$ \\
\hline Phormidium sp. & $\checkmark$ & $\checkmark$ & $\checkmark$ \\
\hline Pseudanabaena biceps Böcher & $\checkmark$ & & $\checkmark$ \\
\hline Pseudanabaena rosea (Skuja) Anagnostidis & $\checkmark$ & $\checkmark$ & $\checkmark$ \\
\hline Pseudophormidium sp. & & & $\checkmark$ \\
\hline Spirulina sp. & $\checkmark$ & & $\checkmark$ \\
\hline Synechococcus sp. & $\checkmark$ & $\checkmark$ & $\checkmark$ \\
\hline Synechocystis sp. & $\checkmark$ & $\checkmark$ & $\checkmark$ \\
\hline Tychonema sp. & & $\checkmark$ & $\checkmark$ \\
\hline Total number of Cyanophyceae species & 12 & 14 & 18 \\
\hline \multicolumn{4}{|l|}{ BACILLARIOPHYCEAE } \\
\hline Achnanthidium sp. & $\checkmark$ & $\checkmark$ & $\checkmark$ \\
\hline Aulacoseira ambigua (Grunow) Simonsen & $\checkmark$ & $\checkmark$ & $\checkmark$ \\
\hline Cyclotella meneghiniana Kützing & $\checkmark$ & & $\checkmark$ \\
\hline Cyclotella ocellata Pantocsek & & $\checkmark$ & \\
\hline Cymbella cymbiformis Agardh & $\checkmark$ & $\checkmark$ & $\checkmark$ \\
\hline Diploneis sp. & & $\checkmark$ & \\
\hline Encyonopsis microcephala (Grunow) Krammer & $\checkmark$ & $\checkmark$ & \\
\hline Eunotia sp. & $\checkmark$ & & \\
\hline Fallacia sp. & & & $\checkmark$ \\
\hline Fragilaria sp. & $\checkmark$ & & \\
\hline Fragilaria crotonensis Kitton & & $\checkmark$ & $\checkmark$ \\
\hline Gomphonema sp. & & $\checkmark$ & \\
\hline Hantzschia amphioxys (Ehrenberg) Grunow & & $\checkmark$ & \\
\hline Melosira varians C. Agardh & $\checkmark$ & & $\checkmark$ \\
\hline Navicula veneta Kützing & & $\checkmark$ & $\checkmark$ \\
\hline Nitzschia sp. & & $\checkmark$ & \\
\hline Nitzschia amphibia Grunow & & & $\checkmark$ \\
\hline Nitzschia dissipata (Kützing) Grunow & & $\checkmark$ & \\
\hline Nitzschia draveillensis Coste et Ricard & $\checkmark$ & $\checkmark$ & \\
\hline Nitschia palea (Kützing) W.Smith & $\checkmark$ & $\checkmark$ & $\checkmark$ \\
\hline Pinnularia subbrevistriata Krammer & $\checkmark$ & & $\checkmark$ \\
\hline Pseudostaurosira brevistriata (Grunow) D.M.Williams et Round & & $\checkmark$ & \\
\hline Rhopalodia sp. & & & $\checkmark$ \\
\hline Sellaphora seminulum (Grunow) D.G.Mann & $\checkmark$ & $\checkmark$ & \\
\hline Staurosira construens Ehrenberg & $\checkmark$ & $\checkmark$ & \\
\hline Staurosira elliptica (Schumann) D.M. Williams et Round & & $\checkmark$ & \\
\hline
\end{tabular}




\begin{tabular}{|c|c|c|c|}
\hline Staurosira sp. & $\checkmark$ & $\checkmark$ & $\checkmark$ \\
\hline Staurosirella sp. & $\checkmark$ & $\checkmark$ & $\checkmark$ \\
\hline Suriella angusta Kützing & & $\checkmark$ & \\
\hline Synedra tenera W. Smith & $\checkmark$ & $\checkmark$ & $\checkmark$ \\
\hline Tabellaria flocculosa (Roth) Kützing & $\checkmark$ & & \\
\hline Tryblionella apiculata Gregory & & $\checkmark$ & $\checkmark$ \\
\hline Total number of Bacillariophyceae species & 17 & 23 & 16 \\
\hline \multicolumn{4}{|l|}{ CHLOROPHYCEAE } \\
\hline Ankistrodesmus densus Korshikov & & $\checkmark$ & \\
\hline Ankistrodesmus fusiformis Corda ex Korshikov & & & $\checkmark$ \\
\hline Ankistrodesmus gracilis (Reinsch) Korshikov & $\checkmark$ & $\checkmark$ & \\
\hline Ankistrodesmus spiralis (W.B.Turner) Lemmermann & $\checkmark$ & $\checkmark$ & \\
\hline Bracteacoccus sp. & $\checkmark$ & $\checkmark$ & $\checkmark$ \\
\hline Chaetophora sp. & & & $\checkmark$ \\
\hline Chlorella sp. & $\checkmark$ & $\checkmark$ & $\checkmark$ \\
\hline Chlamydomonas sp. & $\checkmark$ & $\checkmark$ & $\checkmark$ \\
\hline Chlorococcum sp. & $\checkmark$ & $\checkmark$ & $\checkmark$ \\
\hline Chroococcus sp. & $\checkmark$ & $\checkmark$ & \\
\hline Chroomonas sp. & $\checkmark$ & $\checkmark$ & $\checkmark$ \\
\hline Coelastrum sp. & $\checkmark$ & $\checkmark$ & $\checkmark$ \\
\hline Coelospaerium sp. & $\checkmark$ & & \\
\hline Crucigeniella sp. & & $\checkmark$ & $\checkmark$ \\
\hline Dictyosphaerium sp. & & $\checkmark$ & \\
\hline Geminella sp. & & $\checkmark$ & \\
\hline Kirchneriella sp. & $\checkmark$ & & \\
\hline Monoraphidium contortum (Thuret) Komárková-Legnerová & $\checkmark$ & $\checkmark$ & $\checkmark$ \\
\hline Monoraphidium minitum (Nägeli) Komárková-Legnerová & & $\checkmark$ & \\
\hline Monoraphidium pusillum (Printz) Komárková-Legnerová & & $\checkmark$ & \\
\hline Monoraphidium tortile (West et G.S.West) Komárková-Legnerová & $\checkmark$ & & \\
\hline Oocystis sp. & $\checkmark$ & $\checkmark$ & $\checkmark$ \\
\hline Pandorina sp. & & & $\checkmark$ \\
\hline Pediastrum duplex Meyen & $\checkmark$ & $\checkmark$ & $\checkmark$ \\
\hline Pediatrum tetras (Ehrenberg) Ralfs & $\checkmark$ & $\checkmark$ & $\checkmark$ \\
\hline Scenedesmus acutus Meyen & $\checkmark$ & $\checkmark$ & \\
\hline Scenedesmus dimorphus (Turpin) Kützing & & $\checkmark$ & $\checkmark$ \\
\hline Scenedesmus dispar Brébisson & & $\checkmark$ & \\
\hline Scenedesmus linearis Komárek & $\checkmark$ & $\checkmark$ & $\checkmark$ \\
\hline Scenedesmus longispina R. Chodat & & $\checkmark$ & \\
\hline Scenedesmus opoliensis P.G. Richter & & $\checkmark$ & \\
\hline Scenedesmus quadricauda Chodat & & $\checkmark$ & \\
\hline Scenedesmus spinosus Chodat & & $\checkmark$ & \\
\hline Scenedesmus tenuispina Chodat & $\checkmark$ & $\checkmark$ & $\checkmark$ \\
\hline Selenastrum sp. & & $\checkmark$ & \\
\hline Sphaerocystis sp. & & $\checkmark$ & \\
\hline Staurastrum sp. & & $\checkmark$ & \\
\hline Tetraedron caudatum (Corda) Hansgirg & & & $\checkmark$ \\
\hline Tetraedron minimum (A. Braun) Hansgirg & $\checkmark$ & & \\
\hline Tetraedron sp. & & $\checkmark$ & \\
\hline Total number of Chlorophyceae species & 20 & 32 & 18 \\
\hline \multicolumn{4}{|l|}{ CRYPTOPHYCEAE } \\
\hline Cryptomonas sp. & $\checkmark$ & & $\checkmark$ \\
\hline Total number of Cryptophyceae species & 1 & $\mathbf{0}$ & 1 \\
\hline
\end{tabular}




\begin{tabular}{|c|c|c|c|}
\hline \multicolumn{4}{|l|}{ CHRYSOPHYCEAE } \\
\hline Paraphysomonas sp. & $\checkmark$ & & \\
\hline Total number of Chrysophyceae species & 1 & $\mathbf{0}$ & $\mathbf{0}$ \\
\hline \multicolumn{4}{|l|}{ EUGLENOPHYCEAE } \\
\hline Euglena sp. & $\checkmark$ & & \\
\hline Total number of Euglenophyceae species & 1 & $\mathbf{0}$ & $\mathbf{0}$ \\
\hline \multicolumn{4}{|l|}{ PRYMNESIOPHYCEAE } \\
\hline Hymenomonas roseola Stein & $\checkmark$ & $\checkmark$ & $\checkmark$ \\
\hline Total number of Prymnesiophyceae species & 1 & 1 & 1 \\
\hline \multicolumn{4}{|l|}{ XANTHOPHYCEAE } \\
\hline Goniochloris sp. & & $\checkmark$ & \\
\hline Total number of Xanthophyceae species & $\mathbf{0}$ & 1 & $\mathbf{0}$ \\
\hline TOTAL PHYTOPLANKTON SPECIES RICHNESS & 53 & 71 & 54 \\
\hline
\end{tabular}

the presence of potentially problematic species scarce in ambient dam waters. These include several cyanoprokaryote genera that can lead to potential water quality problems, namely Anabaena, Leptolyngbya, Phormidium, Synechococcus, Lyngbya, Microcystis, Oscillatoria and Synechocystis.

Figures 2-4 compare the algal classes and Cyanophyceae (cells. $\mathrm{m}^{-1}$ ) in Klerkskraal, Boskop and Potchefstroom dams during the previous survey with that of the current survey. During both surveys the Chrysophyceae was more prominent during the cooler months in all three dams. During the previous survey the Chlorophyceae usually dominated during the warmer months, but during the current survey the Bacillariophyceae were more abundant during summer in all three dams. This tendency is also reflected in the richness of Chlorophyceae, where species numbers generally decreased from the previous to current survey, and in the richness of the Bacillariophyceae, which showed a general increase in species number from the previous to current study (Table 1).

Figure 2 clearly shows that the Chrysophyceae was the dominant algal group in Klerkskraal Dam during the previous survey, but their cell numbers were significantly $(p=0.02)$ lower during the current survey. Cryptophyceae (Table 3 ) were also less abundant $(p=0.03)$ during the current survey than during the previous survey. The decline in concentration of both these groups probably accounts for the significant decrease in the total number of cells observed during the current sampling period. No significant difference was observed between the abundance of any of the other algal classes, including the Cyanophyceae $(p>0.05)$, during the two surveys. During the current period the Bacillariophyceae dominated with an average of 203 cells $\cdot \mathrm{ml}^{-1}$. The abundance of Cyanophyceae, often indicative of nutrient pollution levels, remained low during both study periods.

There were no significant changes in the algal and cyanoprokaryote concentrations of Boskop Dam (Fig. 3), except for a significant increase in the Bacillariophyceae cells $(p=0.01)$ which dominated during the current period. Although there was a drastic decline in the numbers of the Chrysophyceae this was not statistically significant $(p=0.6)$. However, species number of Cyanophyceae increased from 3 to 8 , with new genera, including bloom-forming Cylindrospermopsis and Microcystis, appearing during the current survey.

No significant change ( $p>0.05)$ was evident in the concentration of the algal or cyanoprokaryote groups found in Potchefstroom Dam (Fig. 4), where the Bacillariophyceae was also the dominant algal group during the current survey with an average of 316 cells $\cdot \mathrm{m}^{-1}$.

Overall, in the three dams, both total algal and cyanoprokaryote concentrations (cells. $\mathrm{l}^{-1}$ ) were much lower during the second study period (2010-2011) than during the previous survey.

\section{Environmental factors and multivariate analysis}

The data used in statistical models for multivariate analyses from the Klerkskraal, Boskop and Potchefstroom dams are shown in Figs. 5 to 7. An indirect linear gradient analysis, the principal component analysis (PCA), was used as an investigative tool to determine relationships between the different water quality variables (with the ranges for these variables summarised in Table 3).

The results of the PCA ordination plot for Klerkskraal Dam (Fig. 5) indicate that the first axis explains $99 \%$ of the variance in the data. This is probably due to the significant increase $(p<0.05)$ in conductivity, from an average value of $236 \mu \mathrm{S} \cdot \mathrm{cm}^{-1}$ during the previous survey to $365 \mu \mathrm{S} \cdot \mathrm{cm}^{-1}$ during the current survey. Conductivity was a major driver in the system which can also be inferred from the length of the vector. The average orthophosphate and ammonium concentrations increased from $10 \mu \mathrm{g} \cdot \ell^{-1}$ to $110 \mu \mathrm{g} \cdot \ell^{-1}$ and $30 \mu \mathrm{g} \cdot \ell^{-1}$ to $110 \mu \mathrm{g} \cdot \ell^{-1}$, respectively, while the dissolved oxygen decreased significantly $(p<0.05)$ from the previous survey $\left(8.45 \mathrm{mg} \cdot \ell^{-1}\right)$ to the current survey $\left(5.06 \mathrm{mg} \cdot \ell^{-1}\right)$.

The same tendency seen in Klerkskraal Dam was also observed in Boskop Dam (Fig. 6). Conductivity, ammonium and orthophosphate concentrations increased significantly in Boskop Dam, while dissolved oxygen decreased significantly from $9.01 \mathrm{mg} \cdot \ell^{-1}$ during the previous survey to $7.35 \mathrm{mg} \cdot \ell^{-1}$ during the current survey. The $\mathrm{pH}$ of Boskop decreased significantly $(p<0.05)$ from 8.4 during the previous survey to 8.12 during the current survey. In Fig. 5 the PCA ordination plot for Boskop Dam indicates that the first axis explains 99.98\% of the variance in the data. This is most probably due to the $42 \%$ increase in the average conductivity from $347 \mu \mathrm{S} \cdot \mathrm{cm}^{-1}$ to $595 \mu \mathrm{S} \cdot \mathrm{cm}^{-1}$ and $97 \%$ increase in the average concentration of orthophosphate from $10 \mu \mathrm{g} \cdot \ell^{-1}$ to $200 \mu \mathrm{g} \cdot \ell^{-1}$ from the previous to the current survey. The $\mathrm{pH}$ and oxygen of all the dams were measured during the morning but diurnal fluctuations could influence the data.

The PCA ordination plot for Potchefstroom Dam (Fig. 7) shows that the first axis explains $99.7 \%$ of the variance 

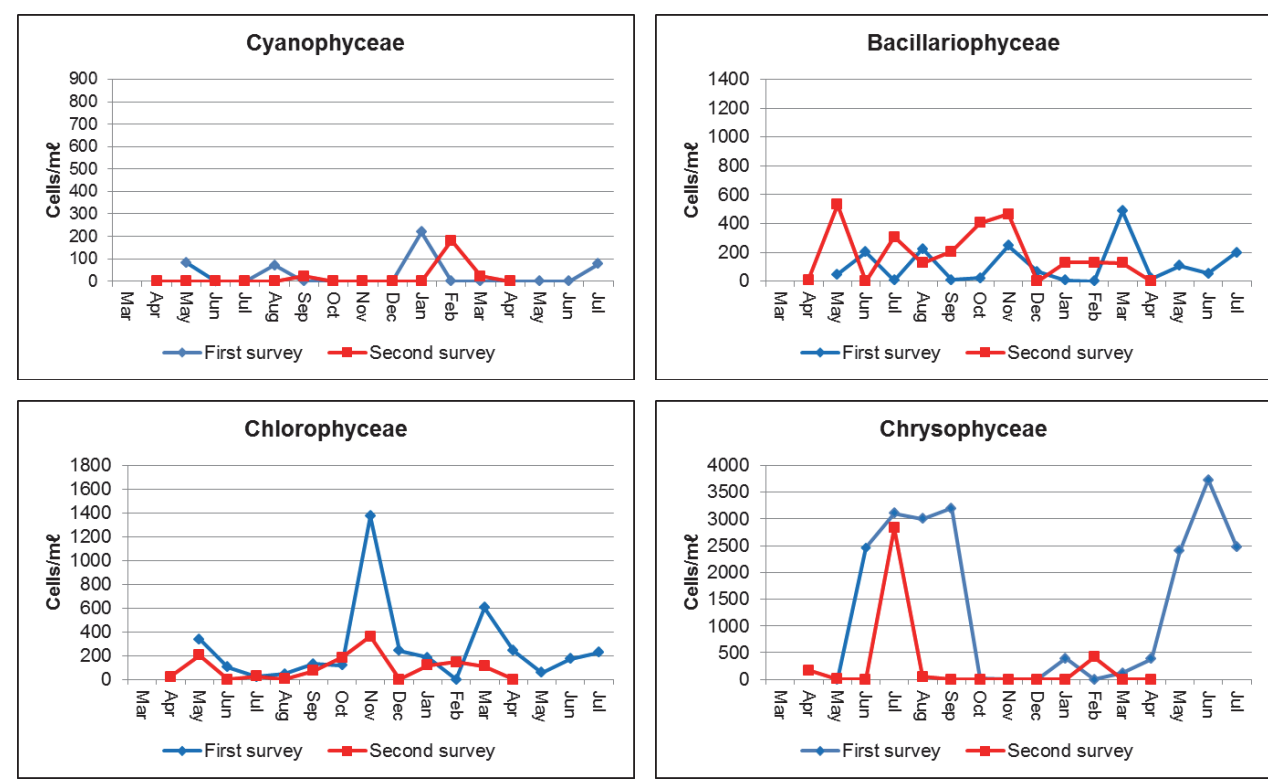

Figure 2

A comparison of the occurrence of specific algal classes and Cyanophyceae in Klerkskraal Dam during 1999-2000 (first survey) and 2010-2011 (second survey)
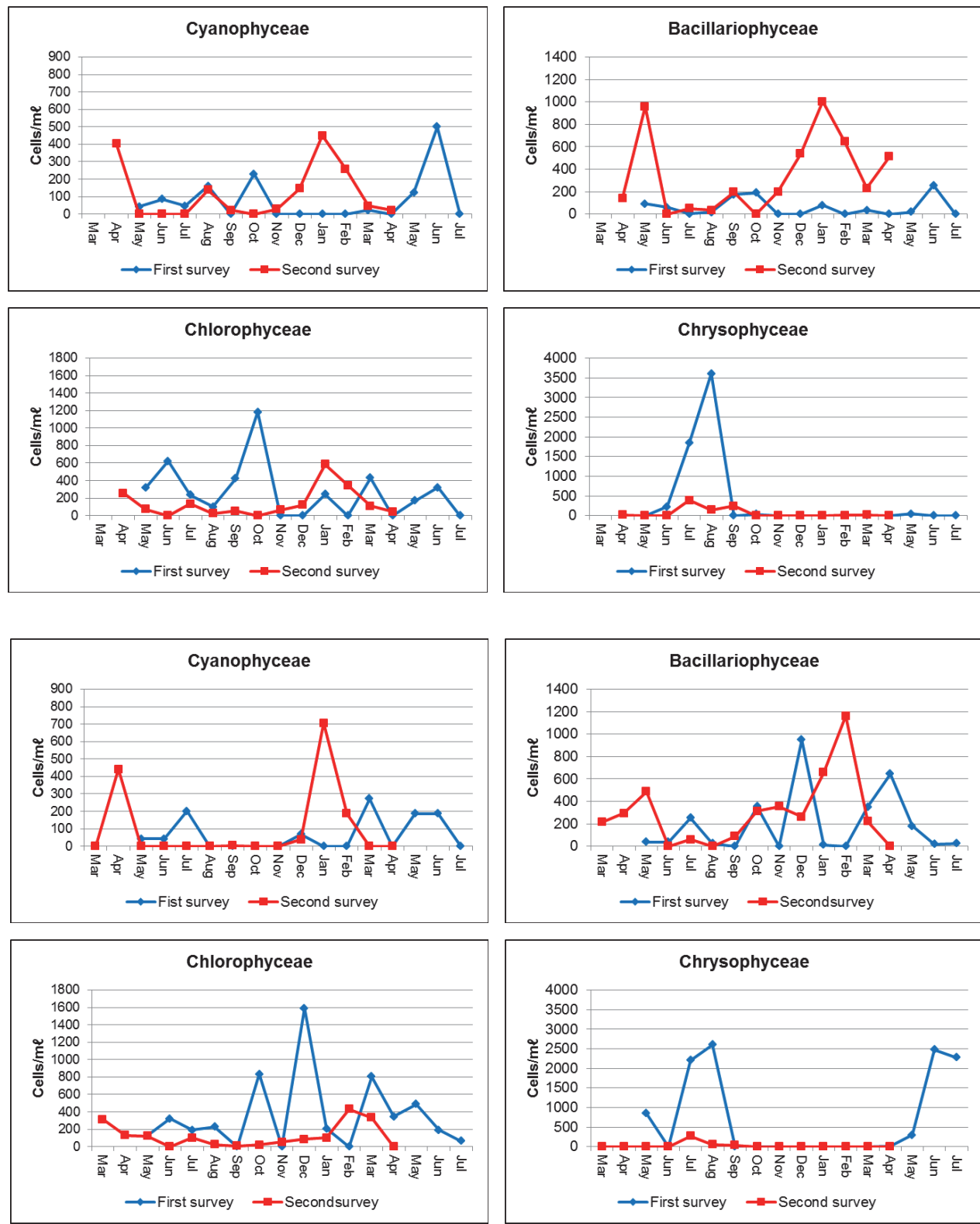

Figure 3

A comparison of the occurrence of specific algal classes and Cyanophyceae in Boskop Dam during 1999-2000 (first survey) and 2010-2011 (second survey)

\section{Figure 4}

A comparison of the occurrence of specific algal classes and Cyanophyceae in Potchefstroom Dam during 1999-2000 (first survey) and 2010-2011 (second survey) 

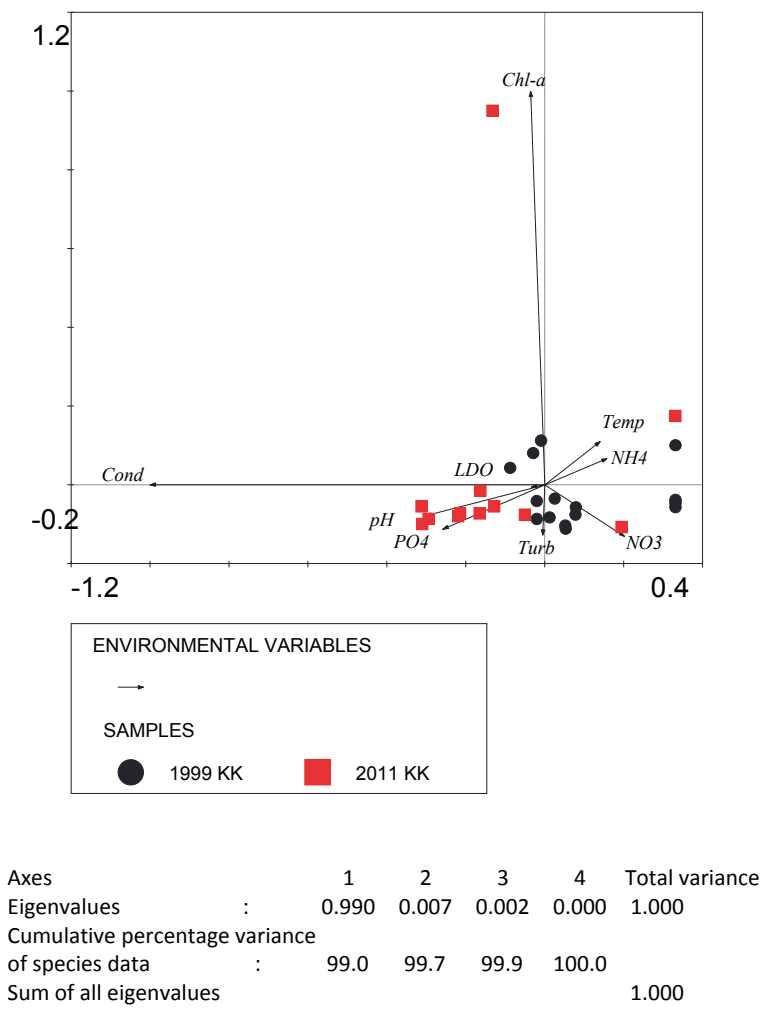

Figure 5

A PCA of the environmental variables of Klerkskraal Dam (KK) measured during the 1999-2000 study period as well as those measured during the 2010-2011 study period
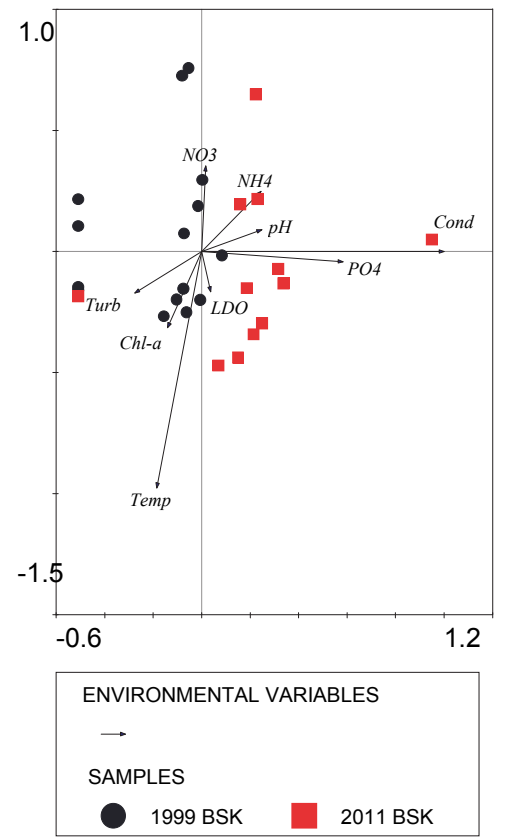

\footnotetext{
$\begin{array}{lccccc}\text { Axes } & 1 & 2 & 3 & 4 & \text { Total variance }\end{array}$ $\begin{array}{lllllll}\text { Eigenvalues } & : & 0.998 & 0.001 & 0.000 & 0.000 & 1.000 \\ \text { Cumulative percentage variance } & & & & & \end{array}$ $\begin{array}{llllll}\text { of species data } & : & 99.8 & 99.9 & 100.0 & 100.0\end{array}$ Sum of all eigenvalues 1.000

Figure 6

A PCA of the environmental variables of Boskop Dam (BSK) measured during the 1999-2000 study period as well as those measured during the 2010-2011 study period
}

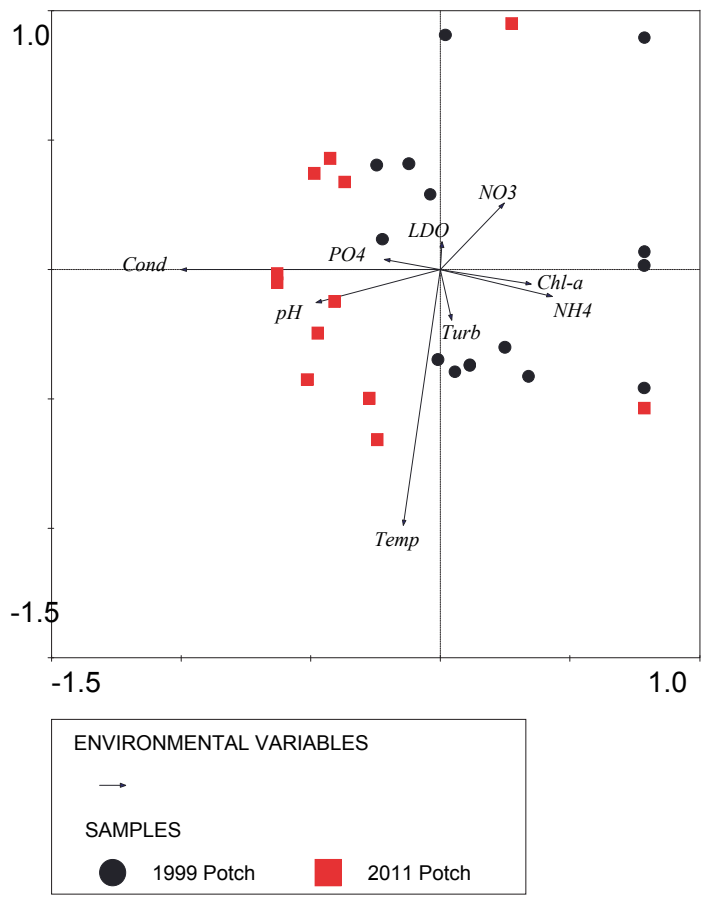

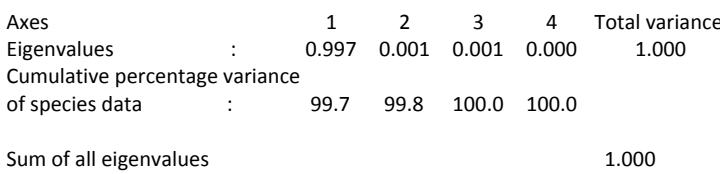

Figure 7

A PCA of the environmental variables of Potchefstroom Dam (Potch) measured during the 1999-2000 study period as well as those measured during the 2010-2011 study period.

in the data. Once again the differences in conductivity and orthophosphate were the most important, with average values that increased from $348 \mu \mathrm{S} \cdot \mathrm{cm}^{-1}$ and $10 \mu \mathrm{g} \cdot \ell^{-1}$ during the previous survey to $573 \mu \mathrm{S} \cdot \mathrm{cm}^{-1}$ and $200 \mu \mathrm{g} \cdot \ell^{-1}$ during the current survey respectively.

It is puzzling that the chlorophyll- $a$ concentrations of the three dams did not change significantly during the decade despite significant increases in orthophosphate concentrations, alongside paradoxical reductions in average cell concentrations. The average concentration of all algae and cyanoprokaryota decreased from 2447 to 629 cells.m $\ell^{-1}$ for Klerkskraal Dam; from 828 to 680 cells $\cdot \ell^{-1}$ for Boskop Dam and from 1462 to 544 cells $\cdot \mathrm{m}^{-1}$ for Potchefstroom Dam. This decrease is largely due to the decrease in the number of Dinobryon cells. Chrysophyceae such as Dinobryon species are widely recognised as mixotrophs (Bellinger and Sigee, 2010) that can supplement nutrients in an oligotrophic environment by consuming bacteria (Holen and Boraas, 1995). Lewitus and Caron (1991) suggested that heterotrophic nutrition ensues at the expense of photosynthetic capabilities and a high probability of the loss of chloroplast function (Holen and Boraas, 1995). Therefore, it is possible that the chlorophyll- $a$ content per cell of Dinobryon is lower than the chlorophyll- $a$ content per Bacillariophyceae cell (dominating during the current survey) accounting for the stable chlorophyll- $a$ concentration. Myers and Graham (1956) found that Poterioochromonas malhamensis (Chrysophyceae) has a lower concentration of cellular chlorophyll in comparison with similar-sized algae. 


\begin{tabular}{|c|c|c|c|c|c|c|c|c|c|c|c|c|c|c|}
\hline & Descrip & tive statisti & cs for $v$ & iables & neasure & in the & $\begin{array}{l}\text { BLE } 3 \\
\text { hree da }\end{array}$ & is for $t$ & surve & in 199 & $-2000 a$ & nd 2010 & 2011 & \\
\hline Variable & Survey & Unit & & & & & & escripti & statist & & & & & \\
\hline & & & Mean & Min & Max & SD & Mean & Min & Max & SD & Mean & Min & Max & SD \\
\hline & & & & Bosk & o Dam & & & otchefs & oom Da & & & Klerkskr & aal Dam & \\
\hline Chl $a$ & 1999-00 & $\mu g \cdot \ell^{-1}$ & 5.4 & 0.57 & 11.5 & 3.91 & 10.9 & 1.59 & 22.9 & 7.21 & 5.52 & 0.29 & 14.14 & 4.79 \\
\hline & 2010-11 & & 7.2 & 0.10 & 18 & 5.89 & 6.72 & 0.10 & 25 & 7.83 & 9.36 & 0.1 & 67 & 18.7 \\
\hline $\mathrm{DO}$ & 1999-00 & $\mathrm{mg} \cdot \ell^{-1}$ & 9 & 7 & 12.8 & 1.44 & 9.1 & 7.42 & 11.61 & 1.29 & 8.45 & 6.82 & 13.58 & 1.73 \\
\hline & $2010-11$ & & 7.4 & 3.66 & 10.6 & 2.30 & 70.2 & 52 & 96 & 15.42 & 5.06 & 0 & 10 & 3.47 \\
\hline Temp & 1999-00 & ${ }^{\circ} \mathrm{C}$ & 18.9 & 10.6 & 25.5 & 5.13 & 19.9 & 10.9 & 23.6 & 4.75 & 18.2 & 9.8 & 23.9 & 4.72 \\
\hline & 2010-11 & & 19.3 & 10.9 & 27.8 & 5.72 & 19.87 & 11.05 & 29.5 & 5.95 & 18.8 & 10.4 & 25.4 & 5.12 \\
\hline Turb & 1999-00 & NTU & 2.2 & 1.30 & 3.46 & 0.65 & 3.87 & 1.3 & 8 & 2.08 & 2.22 & 0.8 & 4.1 & 0.94 \\
\hline & $2010-11$ & & 2.5 & 0 & 4.80 & 2.32 & 3.25 & 0 & 6.9 & 3.03 & 2.17 & 0 & 5.1 & 2.42 \\
\hline Cond & 1999-00 & $\mu \mathrm{S} \cdot \mathrm{cm}^{-1}$ & 347 & 266 & 447 & 47.9 & 348.6 & 206 & 476 & 86.9 & 236 & 187 & 310 & 37.9 \\
\hline & $2010-11$ & & 595.7 & 436 & 1101 & 176.9 & 537.8 & 236 & 653 & 115 & 364.8 & 101 & 476 & 108 \\
\hline $\mathrm{pH}$ & 1999-00 & & 8.4 & 7.98 & 8.81 & 0.27 & 8.46 & 7.78 & 8.90 & 0.33 & 8.25 & 7.50 & 8.79 & 0.41 \\
\hline & $2010-11$ & & 8.12 & 7.65 & 8.67 & 0.35 & 8.30 & 7.7 & 8.83 & 0.37 & 8.2 & 7.4 & 8.9 & 0.42 \\
\hline $\mathrm{PO}_{4}$ & 1999-00 & $\mathrm{mg} \cdot \ell^{-1}$ & 0.01 & 0 & 0.03 & 0.01 & 0.01 & 0 & 0.03 & 0.01 & 0.01 & 0.0001 & 0.03 & 0.01 \\
\hline & 2010-11 & & 0.20 & 0.05 & 0.99 & 0.28 & 0.20 & 0.03 & 0.95 & 0.28 & 0.11 & 0 & 0.3 & 0.09 \\
\hline $\mathrm{NO}_{3}$ & 1999-00 & $\mathrm{mg} \cdot \ell^{-1}$ & 0.38 & 0 & 1.9 & 0.58 & 0.15 & 0 & 1.29 & 0.33 & 0.13 & 0 & 0.72 & 0.19 \\
\hline & $2010-11$ & & 0.32 & 0 & 1.30 & 0.39 & 0.14 & 0 & 0.40 & 0.15 & 0.12 & 0 & 0.4 & 0.14 \\
\hline $\mathrm{NH}_{4}$ & 1999-00 & $\mathrm{mg} \cdot \mathrm{l}^{-1}$ & 0.03 & 0 & 0.09 & 0.03 & 0.06 & 0 & 0.41 & 0.11 & 0.03 & 0 & 0.1 & 0.03 \\
\hline & $2010-11$ & & 0.06 & 0.02 & 0.10 & 0.03 & 0.09 & 0 & 0.28 & 0.08 & 0.11 & 0 & 0.5 & 0.13 \\
\hline Cyano & 1999-00 & cells $\cdot \mathrm{m} \ell^{-1}$ & 81 & 0 & 501 & 136 & 68 & 0 & 272 & 98 & 30 & 0 & 220 & 61 \\
\hline & $2010-11$ & & 92 & 0 & 449 & 138 & 788 & 0 & 706 & 205 & 19 & 0 & 180 & 52 \\
\hline Bacil & 1999-00 & cells $\cdot \mathrm{m}^{-1}$ & 61 & 0 & 255 & 82 & 204 & 0 & 950 & 289 & 113 & 0 & 486 & 136 \\
\hline & $2010-11$ & & 364 & 0 & 1001 & 361 & 300 & 0 & 116 & 342 & 202 & 0 & 532 & 185 \\
\hline Chloro & 1999-00 & cells $\cdot m \ell^{-1}$ & 270 & 0 & 1181 & 317 & 376 & 0 & 1590 & 441 & 259 & 0 & 1373 & 343 \\
\hline & 2010-11 & & 129 & 0 & 583 & 170 & 106 & 0 & 429 & 136 & 108 & 0 & 360 & 110 \\
\hline Crypto & 1999-00 & cells $\cdot \mathrm{m}^{-1}$ & 21 & 0 & 126 & 37 & 11 & 0 & 103 & 28 & 23 & 0 & 149 & 38 \\
\hline & $2010-11$ & & 3 & 0 & 14 & 6 & 8 & 0 & 3 & 11 & 6 & 0 & 23 & 9 \\
\hline Chryso & 1999-00 & cells $\cdot m \ell^{-1}$ & 383 & 0 & 3604 & 1008 & 704 & 0 & 2603 & 1111 & 1419 & 0 & 3730 & 1485 \\
\hline & $2010-11$ & & 67 & 0 & 386 & 127 & 30 & 0 & 266 & 76 & 276 & 0 & 2831 & 814 \\
\hline Dino & 1999-00 & cells $\cdot m \ell^{-1}$ & 7 & 0 & 34 & 10 & 4 & 0 & 26 & 7 & 3 & 0 & 17 & 6 \\
\hline & 2010-11 & & 13 & 0 & 109 & 31 & 9 & 0 & 61 & 19 & 2 & 0 & 20 & 6 \\
\hline Eugleno & 1999-00 & cells $\cdot \mathrm{m}^{-1}$ & 5 & 0 & 51 & 14 & 34 & 0 & 389 & 103 & 13 & 0 & 69 & 18 \\
\hline & 2010-11 & & 11 & 0 & 46 & 15 & 14 & 0 & 74 & 26 & 6 & 0 & 32 & 11 \\
\hline Tcells & 1999-00 & cells $\cdot \mathrm{m}^{-1}$ & 828 & 0 & 3907 & 1070 & 1462 & 0 & 2891 & 1123 & 2447 & 0 & 11357 & 2773 \\
\hline & 2010-11 & & 680 & 0 & 2065 & 589 & 544 & 0 & 1785 & 550 & 629 & 0 & 3180 & 868 \\
\hline
\end{tabular}

According to Oliver et al. (1999) the correlation between chlorophyll and total phosphorus concentrations has been described for a broad range of lakes and is surprisingly congruent for one-factor dependency, but is not suitable in environments where the biomass yield is limited by light or by nutrients other than phosphorus. Environmental factors of importance in modifying the total phosphorus-chlorophyll models are light availability and the supply of nutrients from sources such as bottom sediments (Oliver et al. 1999; Nicholls and Dillon, 1978; Walker, 1995). We did not measure the total phosphate or the total nitrogen but nitrogen limitation could have played a role. The supply of nutrient from sediments is also an issue that is being addressed in ongoing studies in the Mooi River System.

\section{DISCUSSION}

By virtue of their high reproductive rates, algae can respond rapidly to natural and/or anthropogenic changes in environmental conditions (Sharov, 2008). Accordingly, they can serve as valuable bio-indicators of water body health. Dominant genera in algal groupings change not only spatially but also seasonally, as physical, chemical and biological conditions in a water body change (Wetzel, 2001). In addition to seasonal changes in the three reservoirs there was also a change in the algal community from Chrysophyceae dominance during the previous study period to Bacillariophyceae dominance during the current study period. According to Bellinger and Sigee (2010), Chrysophyceae occur in low-nutrient lakes and are considered by some authors as an indicator of oligotrophy (Rawson, 2012). The replacement of Chrysophyceae species by Bacillariophyceae species, such as Melosira varians, Cyclotella meneghiniana and Aulacoseira granulata, that are typical of eutrophic impoundments, was more pronounced in Boskop Dam than in any of the other dams. High numbers of Fragilaria ulna present in mesotrophic to eutrophic, alkaline water (Taylor et al., 2007a) were also observed in Boskop Dam, 
indicating a decrease in the water quality of this dam over time The diatom Asterionella formosa was not found during the current survey in the Boskop or Potchefstroom dams. This species is generally found in the plankton of mesotrophic dams (Taylor et al., 2007a) and appears to have been replaced by the nutrienttolerant taxa mentioned above.

Diatoma vulgaris, a diatom species indicative of hard water, with elevated nutrient levels (Janse van Vuuren et al., 2006), was absent in all three dams during the previous survey. Its presence in all of the dams during the current survey (Table 1) is indicative of enrichment over the past decade. Walsh and Wepener (2009) showed that agricultural enrichment favours the presence of this species, as illustrated by its high abundance in Bloemhof Dam, an irrigated agricultural region. According to Taylor et al. (2005) and Hill et al. (2001), environmental preferences for $D$. vulgaris include conductivity levels of 100 to $500 \mu \mathrm{S} \cdot \mathrm{cm}^{-1}$ and mesotrophic to eutrophic conditions. Results from this study indicated that conductivity was one environmental variable that increased most markedly (by $42 \%$ ) from the previous to current survey. South African studies by Taylor et al. (2007b) linked D. vulgaris specifically to freshwaters with elevated levels of phosphatephosphorus. Our results showed that nutrient concentrations (phosphorus and ammonium) increased over the decade. In Boskop Dam the phosphorus concentration increased by $97 \%$. Increasing nutrient concentrations, together with high conductivity values, probably triggered the occurrence of this species.

The number of Cyanophyceae species identified during the previous and current surveys stayed the same in Klerkskraal Dam and more or less the same in Potchefstroom Dam (Table 1). However, in Boskop Dam, Cyanophyceae increased both in species richness ( 3 to 8 ) and average numerical abundance ( 81 to 93 cells $\cdot \mathrm{m}^{-1}$ ). The numerical increase resulted from an increase in the abundance of potentially harmful species, such as Microcystis sp., Oscillatoria sp. and Cylindrospermopsis raciborskii. The potential for these organisms to become problematic under changing conditions is high, as even more Cyanophyceae species were observed in the enriched medium than during the enumeration of the samples (Table 2). Although the Chlorophyceae was the most species-rich algal class (Tables 1 and 2), there was a decline in the species richness of the Chlorophyceae in all three dams (Table 1) from the previous to the current survey.

While only one species of Chrysophyceae (Dinobryon) was recorded in lake water samples (Table 1), additional chrysophytes appeared in enriched samples (Table 2), namely, Paraphysomonas sp. in Klerkskraal Dam, and an unidentified naked colonial species that occurred in all three dams (not listed).

Some of the algae that are scarce (or absent) in dam water samples were found in enriched samples. These algae include Geminella sp., Paraphysomonas sp. as well as Hymenomonas roseola. Geminella sp. is classified under the Chlorophyceae and has turpin filaments that consist of cells in a separate, but loose, linear arrangement. Cells are longer than broad, cylindrical with round apices, with a parietal chloroplast and usually one pyrenoid. This alga was only found in the enriched sample from Boskop Dam. Hymenomonas roseola Stein 1878 is a freshwater coccolithophorid classified under the Class Prymnesiophyceae (Stang, 2004). According to John et al. (2002), the motile cells are ellipsoidal to subspherical and $13-50 \times 10^{-24} \mu \mathrm{m}$ with a long flagellum and a short haptoneme. Coccoliths (scales) are circular to elliptical. This species was found at all the sampling localities. Paraphysomonas sp. De Saedeler belongs to the Chrysophyceae and has a long flimmer flagellum and one short smooth flagellum. Cells are solitary, covered in siliceous scales and lack any chloroplast (Wehr and Sheath, 2003). This alga was only found in the enriched samples from Klerkskraal Dam.

Conductivity, as well as orthophosphate and ammonium levels of all three dams increased between the previous and current survey, while the dissolved oxygen concentration decreased (in line with the lower algal concentration). The $\mathrm{pH}$ decreased significantly in Boskop and Potchefstroom dams and has the potential to increase the bioavailability and toxicity of metals (Wetzel, 2001) in the water bodies. Metals most likely to have increased detrimental environmental effects, as a result of lowered $\mathrm{pH}$, are silver, aluminium, cadmium, cobalt, copper, mercury, manganese, nickel, lead and zinc (DWAF, 1996). However, as these problems only emerge below $\mathrm{pH} 7$, there would have to be a significant and constant source of acid pollution sufficient to exceed the Mooi River system's naturally high buffering capacity, related to its hard dolomite catchment. The average $\mathrm{pH}$ for all three dams was higher than 8 for both study periods and can cause the conversion of ammonium ions to the highly toxic un-ionized ammonia (DWAF, 1996). The ammonium ion is not toxic to aquatic biota, but contributes to eutrophication (DWAF, 1996).

\section{CONCLUSIONS}

An overview of Klerkskraal, Boskop and Potchefstroom dams showed that both total algal and cyanoprokaryote concentrations were lower during the current survey (2010-2011), suggesting improved ecosystem health. Therefore, these dams can still be classified as oligo- to mesotrophic (using criteria of Van Ginkel, 2002). However, there are indications, such as increasing conductivity and nutrient concentrations (particularly phosphate), that the trophic status, especially for Boskop Dam, is changing. A shift in the main drivers of these ecosystems is reflected in the change from a Chrysophyte-dominated community to a community where the Bacillariophyceae, particularly those species common in eutrophic impoundments, are dominant. Enrichment of samples under culture conditions also revealed the presence of problem species such as Cylindrospermopsis and Microcystis, that are likely to proliferate if these reservoirs experience further increases in nutrient concentrations, thereby decreasing the water quality of the Mooi River system.

\section{REFERENCES}

ANNANDALE E and NEALER E (2011) Exploring aspects of the water history of Potchefstroom region and the local management of it. New Contree 62. URL: http://dspace.nwu.ac.za/bitstream/ handle/10394/6611/No 62(2011) Annandale E \%26 Nealer E. pdf? sequence $=1$ (Accessed April 2013).

BELLINGER EG and SIGEE DC (2010) Freshwater algae: Identification and Use as Bioindicators. Wiley-Blackwell, Oxford, UK. 271 pp.

COETZEE H, WINDE F and WADE PW (2006) An assessment of sources, pathways, mechanisms and risks of current and potential future pollution of water and sediments in gold-mining areas of the Wonderfonteinspruit catchment. WRC Report No. 1214/1/06. Water Research Commission, Pretoria. 266 pp.

CROASDALE H, FLINT EA and RACINE MM (1994) Flora of New Zealand: freshwater algae, Chlorophyta, desmids with ecological comments on their habitats Volume 111. Manaaki Whenua Press, Lincoln, New Zealand. 218 pp. 
CURRIE SL (2001) The implementation of an environmental decisionmaking support system: the Mooi River Catchment as an case study. PhD dissertation, Potchefstroom University for Christian Higher Education, Potchefstroom. 152 pp.

DE LA REY PA, TAYLOR JC, LAAS A, VAN RENSBURG L and VOSLOO A (2004) Determining the possible application value of diatoms as indicators of general water quality: a comparison with SASS5. Water SA 30 (3) 325-332.

DWAF (DEPARTMENT OF WATER AFFAIRS AND FORESTRY, SOUTH AFRICA) (1996) South African Water Quality Guidelines, Volume 7 Aquatic Ecosystems. 161pp. URL: http://www.dwaf.gov. $\mathrm{za} /$ (Accessed October 2010).

ETTL H, GäRTNER G, HEYNIG H, MOLLENHAUER D, KOMáREK J and AGNOSTIDIS K (1999) Susswasser von Mittleeuropa: Cyanoprokaryota, Teil 1: Chroococcales. Gustav Fisher Verlag, Jena, Germany. 548 pp.

HILL BH, STEVENSON RJ, PAN Y, HERLIHY AT, KAUFMANN PR and JOHNSON CB (2001) Comparison of correlations between environmental characteristics and stream diatom assemblages characterised at genus and species levels. J. N. Am. Benthol. Soc. 20 (2) 299-310.

HINDAK F (2008) Atlas of Cyanophytes. Veda, Bratislava. 253 pp.

HOLEN DA and BORAAS ME (1995) Mixotrophy in Chrysophytes In: Sandgren CD, Smol JP and Kristiansen J (eds.) Chrysophyte Algae: Ecology, Phylogeny and Development. Cambridge University Press, Cambridge. 403 pp.

HUBER-PESTALOZZI G (1961) Das Phytoplankton des Susswassers: Systematik und Biologie Tiel 5: Chlorophyceae (Grünalgen). Ordnung: Volvocales. E Schweizerbart'sche Verlagbuchhandlung, Stuttgart. 1043 pp.

JANSE VAN VUUREN S, TAYLOR JC, GERBER A and VAN GINKEL C (2006) Easy Identification of the Most Common Freshwater Algae. A Guide for the Identification of Microscopic Algae in South African Freshwaters. North-West University and Department of Water Affairs and Forestry. 200 pp.

JOHN DM, WHITTON BA and BROOK AJ (2002) Freshwater Algal Flora of the British Isles: A Guide to Freshwater and Terrestrial Algae. Cambridge University Press, Cambridge. 702 pp.

KAZI TG, ARAIN MB, JAMALI MK, JALBANI N, AFRIDI HI, SARFRAZ RA, BAIG JA and SHAH-ABDUL Q (2009) Assessment of water quality of polluted lake using multivariate statistical techniques: A case study. Ecotoxicol. Environ. Saf. 72 301-309.

KOMÁREK J and ANAGNOSTIDIS K (2005) Cyanoprokaryota: Oscillatoriales. In: Büdel B, Gärtner G, Krienitz L and Schagerl M (eds.) Freshwater Flora of Central Europe, Part 2. Spektrum Akademischer Verlag, Jena, Germany. 758 pp.

KRÜGER GHJ (1978) The effect of physio-chemical factors on the growth relevant to the mass culture of Microcystis under sterile conditions. PhD dissertation, University of the Orange Free State. 134 pp.

LEWITUS AJ and CARON DA (1991) Physiological responses of photoflagellates to dissolved organic substrate additions: Dominant role of autotrophic nutrition in Pyrenomonas salvina (Cryptophyceae). Plant Cell Physiol. 32 (6) 791-801.

MOGAKABE E (programme manager) (2004) Trophic status of South African impoundments. URL: http://www.dwaf.gov.za/iwqs/ eutrophication/NEMP/nempdam.htm (Accessed October 2013).

MYERS J and GRAHAM J (1959) The role of photosynthesis in the physiology of Ochromonas. J. Cell. Comp. Physiol. 47 (3) 397-414.

NICHOLLS KH and DILLON PJ (1978) An evaluation of phosphoruschlorophyll-phytoplankton relationships for lakes. Int. Rev. Hydrobiol. Hydrograph. 63 (2) 141-154.

OLIVER RL, HART BT, OLEY J, GRACE M, REES C and CAITCHEON G (2000) The Darling River: Algal growth and the cycling and sources of nutrients. Project M386. Murray-Darling
Basin Commission, Canberra City, Australia. 307 pp. URL: http://www2.mdbc.gov.au/_data/page/307/darling_algal_growthwhole.pdf (Accessed May 2013).

O'NIEL JM, DAVIS TW, BURFORD MA and GROBLER CJ (2012) The rise of harmful cyanobacterial blooms: the potential roles of eutrophication and climate change. Harmful Algae 14 313-334.

OYADOMARI J (2001) Keweenaw algae. URL: www.keweenawalgae. mtu.edu (Accessed October 2011).

RAWSON DS (2012) Algal indicators of trophic lake types. URL: www. aslo.org (Accessed August 2012).

SARTORY DP (1982) Spectrophotometric analysis of chlorophyll $a$ in freshwater phytoplankton. Technical Report TR 115. Hydrological Research Institute, Department of Environmental Affairs, Pretoria. $163 \mathrm{pp}$.

SHAROV AN (2008) Phytoplankton as an indicator in estimating long-term changes in the water quality of large lakes. Water Resour. 35 (6) 668-673.

STANG D (2004) Hymenomonas roseola. URL: http://zipcodezoo.com/ Chromista/H/Hymenomonas roseola/\#Description (Accessed October 2011).

STEINBERG CEW and HARTMAN HM (1988) Planktonic bloomforming cyanobacteria and the eutrophication of lakes and rivers. Freshwater Biol. 20 (2) 279-287.

SWANEPOEL A, DU PREEZ H, SCHOEMAN C, JANSE VAN VUUREN S and SUNDRAM A (2008) Condensed laboratory methods for monitoring phytoplankton, including cyanobacteria, in South African freshwaters. WRC Report No. TT 323/08. Water Research Commission, Pretoria. 108 pp.

TAYLOR JC, HARDING WR and ARCHIBALD CGM (2007a) An illustrated guide to some common diatom species from South Africa. WRC Report No. TT 282/07. Water Research Commission, Pretoria. 178 pp.

TAYLOR JC, HARDING WR, ARCHIBALD CGM and VAN RENSBURG L (2005) Diatoms as indicators of water quality in the Jukskei-Crocodile River system in 1956 and 1957, a re-analysis of diatom count data generated by BJ Cholnoky. Water SA 31 (2) 1-10.

TAYLOR JC, PRYGIEL J, VOSLOO, DE LA REY A and VAN RENSBURG L (2007b) Can diatom-based pollution indices be used for biomonitoring in South Africa? A case study of the Crocodile West and Marico water management area. Hydrobiologica 592 455-464.

TER BRAAK CJF and SMILAUER P (1998) CANOCO Reference Manual and User's Guide to Canoco for Windows: Software for Canonical Community Ordination (version 4). Microcomputer Power, Ithaca, NY USA. 352 pp.

UTERMÖHL H (1958) Zur Vervollkomnung der quantitativen Phytoplankton-Methodik. Mitteilungen Internationalen Vereinigung für Limnologie 9 1-38.

VAN GINKEL C (2002) Trophic status assessment. Institute for Water Quality Studies, Department of Water Affairs and Forestry, Pretoria. 17 pp. URL: http://www.dwaf.gov.za/iwqs/eutrophication/NEMP/default.htm (Accessed April 2011).

WALKER WW and HAVENS KE (1995) Relating algal bloom frequencies to phosphorus concentrations in Lake Okeechobee. Lake Reserve Manage. 11 (1) 77-83. URL: http://wwwalker.net/pdf/ chlaokee.pdf (Accessed April 2013).

WALSH G and WEPENER V (2009) The influence of land use on water quality and diatom community structures in urban and agriculturally stressed rivers. Water SA 35 (5) 579-594.

WEHR JD and SHEATH RG (2003) Freshwater Algae of North America, Ecology and Classification. Academic Press, London. 918 pp.

WETZEL RG (2001) Limnology ( $3^{\text {rd }}$ edn.). Elsevier Academic Press, San Diego. 1006 pp.

http://dx.doi.org/10.4314/wsa.v39i5.16

Available on website http://www.wrc.org.za

ISSN 0378-4738 (Print) = Water SA Vol. 39 No. 5 October 2013

ISSN 1816-7950 (On-line) = Water SA Vol. 39 No. 5 October 2013 
http://dx.doi.org/10.4314/wsa.v39i5.16 Available on website http://www.wrc.org.za

ISSN 0378-4738 (Print) = Water SA Vol. 39 No. 5 October 2013 ISSN 1816-7950 (On-line) = Water SA Vol. 39 No. 5 October 2013 